\section{OC-015 DISEASE PROFILE AND LONG-TERM OUTCOME OF PATIENTS WITH AUTOIMMUNE PANCREATITIS/IGG4 SYSTEMIC DISEASE}

\author{
doi:10.1136/gut.2011.239301.15
}

M THuggett, " K Tang, G J Johnson, A R Hatfield, S P Pereira, G J Webster Department of Gastroenterology, University College Hospital, London, UK

Introduction The diagnostic features, disease course, and management of autoimmune pancreatitis (AIP)/IgG4 systemic disease are still being defined. Here we report long-term data on the largest European cohort of prospectively followed patients.

Methods All patients referred to our centre from 20042010, in whom a diagnosis of AIP was made, were followed prospectively.

Results 52 patients (41 M, $11 \mathrm{~F}$; median age 59 years (range 26-85)) were included, with a median follow-up from diagnosis of 32 months (range 0-76). 40 patients were jaundiced at presentation $(77 \%)$ and 7 patients (14\%) had undergone surgery for suspected pancreaticobiliary cancer. HISORt criteria were fulfilled in 46 (89\%) patients. Serum IgG4 was raised in 35 patients (67\%). Histological samples were taken in 49 patients. Tissue IgG4 was raised ( $>10$ cells/hpf) in 29 patients (59\% of those who had at least one biopsy). 17 patients had associated autoimmune disease (7 Sjögren's, 7 thyroid disease, 4 IBD and 1 vitiligo). 41 patients (79\%) had extrapancreatic disease: biliary involvement in 37 patients (71\%) (of whom 2 (4\%) developed cirrhosis and 1 (2\%) underwent liver transplantation); retroperitoneal fibrosis in 5 patients $(10 \%)$; renal infiltrates in 5 (10\%); arthropathy/arthralgia in $3(6 \%)$; IgG4-related cerebral involvement in 2 patients (4\%) (encephalitis and pituitary mass); biopsy-proven peripheral lymphadenopathy (submandibular, iliac or axillary) in $3(6 \%)$; extensive small bowel mesenteric involvement in 1; and pulmonary involvement in 1 patient. Exocrine insufficiency occurred in 23 patients (44\%) and diabetes in 17 (33\%). Portal and/or splenic vein thrombosis developed in 7 patients (14\%). 45 patients (87\%) received steroids for a median of 4.6 months (range 0.6-76). A good response to steroids was seen in $43(96 \%)$ of these patients. Disease relapse occurred in 24 (46\%), with a median time to relapse of 4.2 months (range $0-38$ ). Extrapancreatic disease at presentation predicted relapse $(\mathrm{P}=0.0004)$, but elevated serum IgG4 did not $(\mathrm{P}=0.14) .19$ patients $(37 \%)$ received additional immunosuppression (azathioprine $(\mathrm{n}=17)$, mycophenylate ( $\mathrm{n}$ $=1)$, ciclosporin $(n=1)$, methotrexate $(n=1)$, infliximab $(n=1)$ ). All patients who relapsed responded to subsequent treatment. 37 patients required biliary stenting with a mean of 2 stents (range 1-10 stents). Three patients (6\%) died during follow-up, all from probable IgG4-related disease: autoimmune encephalitis, progressive liver disease and hilar cholangiocarcinoma.

Conclusion Long-term follow-up in this large cohort shows that AIP/IgG4 systemic disease may be associated with multiorgan complications, which cause significant morbidity, and a small but significant mortality. Extrapancreatic involvement at presentation predicts a complicated course.

Competing interests None. 